\author{
Nota científica
}

\title{
Nuevos registros de hongos micorrizógenos arbusculares para México
}

\author{
New records of arbuscular mycorrhizal fungi for Mexico \\ Eduardo Chimal-Sánchez ${ }^{\mathrm{a}}$, Noé Manuel Montaño ${ }^{\mathrm{a}, *}$, Sara Lucía Camargo-Ricalde ${ }^{\mathrm{a}}$, \\ Rosalva García-Sánchez ${ }^{\mathrm{b}}$ y Laura Verónica Hernández-Cuevas ${ }^{\mathrm{c}}$ \\ a Departamento de Biología, División de Ciencias Biológicas y de la Salud, Universidad Autónoma Metropolitana-Iztapalapa, 55-535, 09340, \\ Ciudad de México, México \\ ${ }^{\mathrm{b}}$ Unidad de Investigación en Ecología Vegetal, Facultad de Estudios Superiores Zaragoza, Universidad Nacional Autónoma de México, Av. Guelatao 66, \\ Col. Ejército de Oriente, 09230, Ciudad de México, México

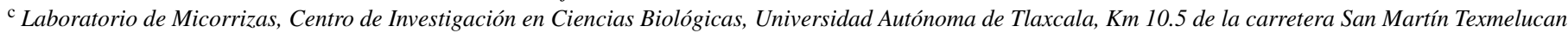 \\ S/N, San Felipe Ixtacuixtla, 90120, Tlaxcala, México \\ Recibido el 17 de abril de 2015; aceptado el 19 de agosto de 2015
}

\begin{abstract}
Resumen
Las especies de hongos micorrizógenos arbusculares Diversispora trimurales, Gigaspora candida y Glomus corymbiforme se registran por vez primera para México. Estos taxa fueron aislados de la rizosfera de Mimosa lacerata, M. luisana, M. polyantha y M. purpusii (LeguminosaeMimosoideae), especies endémicas de México, 2 de las cuales están restringidas al Valle de Tehuacán-Cuicatlán, Puebla y Oaxaca. Los ejemplares revisados se describen en extenso y se comparan con las especies de hongos micorrizógenos arbusculares más parecidas.

Derechos Reservados (C) 2016 Universidad Nacional Autónoma de México, Instituto de Biología. Este es un artículo de acceso abierto distribuido bajo los términos de la Licencia Creative Commons CC BY-NC-ND 4.0.
\end{abstract}

Palabras clave: Ecosistemas áridos; Glomeromycota; Mimosa; Tehuacán-Cuicatlán

\begin{abstract} Creative Commons CC License BY-NC-ND 4.0.

Keywords: Arid ecosystems; Glomeromycota; Mimosa; Tehuacán-Cuicatlán

Los hongos micorrizógenos arbusculares (HMA) del Phylum Glomeromycota (Schüßler, Schwarzott y Walker, 2001) se asocian con el $80 \%$ de las plantas terrestres y forman la micorriza arbuscular (Brundrett, 2009). Estos hongos son elementos importantes para la productividad de los ecosistemas (Van der Heijden et al., 1998), en particular de los áridos y
\end{abstract}

The arbuscular mycorrhizal fungal species Diversispora trimurales, Gigaspora candida and Glomus corymbiforme are reported for the first time in Mexico. These taxa were collected from the rhizosphere of Mimosa lacerata, M. luisana, M. polyantha, and M. purpusii (LeguminosaeMimosoideae), endemic species to Mexico, 2 of which are distributed only in the Valley of Tehuacán-Cuicatlán, Puebla and Oaxaca. The revised specimens are described in detail and compared with the most similar species of arbuscular mycorrhizal fungi.

All Rights Reserved (C) 2016 Universidad Nacional Autónoma de México, Instituto de Biología. This is an open access item distributed under the

\footnotetext{
* Autor para correspondencia.

Correo electrónico: nmma@xanum.uam.mx (N.M. Montaño).

La revisión por pares es responsabilidad de la Universidad Nacional Autónoma de México.
}

semiáridos, ya que incrementan la tolerancia a la sequía y favorecen la captación de fósforo y nitrógeno del suelo en las plantas hospederas (Smith, Facelli, Pope y Smith, 2010).

En México se han registrado 101 especies de HMA (MéndezCortés, Marmolejo, Olalde-Portugal, Cantú y Varela, 2012; Montaño et al., 2012), de las cuales el $41 \%$ se ha descrito en ecosistemas áridos y semiáridos. No obstante, la diversidad de HMA se ha evaluado en menos del 7\% de las ca. 6,000 especies de plantas que existen en estos ambientes (Montaño et al., 2012), lo que hace necesario definir la condición micorrícica y la identidad de los HMA en el $93 \%$ de la diversidad florística 
restante, ya que la composición de especies de HMA puede afectar aspectos como la eficiencia de captación de fósforo o la tolerancia a patógenos de la raíz en las plantas hospederas (Maherali y Klironomos, 2007). El reconocimiento preciso de los hongos micorrizógenos arbusculares que se asocian a especies vegetales particulares constituye el primer paso para generar información que, además de contribuir al conocimiento biológico del país, también permita el planteamiento de estrategias adecuadas para la conservación y manejo conjunto de la vegetación y de los HMA en estos ecosistemas.

El Valle de Tehuacán-Cuicatlán, localizado en los estados de Puebla y Oaxaca en México, tiene aproximadamente $10,000 \mathrm{~km}^{2} \mathrm{y}$, en el año 1998 , el $50 \%$ de su territorio fue declarado Reserva de la Biosfera por el gobierno mexicano (Semarnat, 1998). El Valle de Tehuacán-Cuicatlán es un ecosistema semiárido con una alta riqueza de especies $(2,621$ spp.) y endemismos florísticos (13.9\%) (Dávila et al., 2002). En el valle, el género Mimosa L. está representado por 16 especies y 6 variedades, de las cuales el $31 \%$ son endémicas (Martínez-Bernal y Grether, 2006). Estas especies, además de ser elementos dominantes o codominantes dentro de sus comunidades (Camargo-Ricalde, Dhillion y Grether, 2002), aumentan la abundancia de esporas de HMA en su rizosfera al formar islas de recursos, que son un microambiente favorable para la esporulación (Camargo-Ricalde y Dhillion, 2003; CamargoRicalde y Esperón-Rodríguez, 2005); sin embargo, se desconoce si también contribuyen en la riqueza de especies de estos simbiontes. Como parte de un estudio sobre la diversidad de HMA asociados a la rizosfera de 4 especies de Mimosa endémicas de México: Mimosa lacerata Rose, M. polyantha Benth., M. luisana Brandegee y M. purpusii Brandegee, estas 2 últimas leguminosas endémicas del Valle de Tehuacán-Cuicatlán (Martínez-Bernal y Grether, 2006); en este artículo se registran y describen, por primera vez para México, 3 especies de HMA.

En el Valle de Tehuacán-Cuicatlán se establecieron 2 sitios de estudio (S). En el S1 (Chapulco, Puebla, 1841'31"N, $97^{\circ} 24^{\prime} 01^{\prime \prime}$ O; 2,291 m snm) coexisten M. lacerata y M. purpusii; y en el S2 (Coxcatlán, Puebla, 18 15'23.7' N, 9709'03" O; $1,131 \mathrm{~m} \mathrm{snm}$ ) solo coexisten M. luisana y M. polyantha. Durante la estación de lluvias —octubre de 2012 - y la seca —abril de 2013 - se recolectaron $500 \mathrm{~g}$ de suelo, profundidad $0-20 \mathrm{~cm}$, bajo la copa de 10 individuos de cada especie de Mimosa y en 10 áreas abiertas; es decir, sin la influencia de Mimosa u otra especie vegetal - control- Un total de 120 muestras de suelo, 60 por estación, se recolectaron para realizar la extracción de las esporas de HMA por el método de tamizado húmedo y decantación (Gerdemann y Nicolson, 1963), seguido de centrifugación $(1,000 \mathrm{rpm})$ en sacarosa al 60\% (p/v). Las esporas se montaron en preparaciones permanentes con alcohol polivinílico-lacto-glicerol y alcohol polivinílico-lactoglicerol + reactivo de Melzer en proporción 1:1 (INVAM, 2013).

La determinación taxonómica de los HMA se basó en el reconocimiento, comparación y contraste de los caracteres morfológicos de sus esporas: a) presencia o ausencia, forma y dimensiones de las hifas de origen; b) tamaño y color; c) número de capas de la pared, arreglo y grosor; d) presencia, posición y forma de ornamentaciones; y e) reacción de las capas con el reactivo de Melzer (INVAM, 2013). Esta información se contrastó con descripciones de las especies de Glomeromycota conocidas y disponibles a través de enlaces en los sitios Web: http://schuessler.userweb.mwn.de/amphylo/ (Schüßler y Walker, 2010) y http://invam.caf.wvu.edu/ (INVAM, 2013), así como en la literatura especializada. Los caracteres de las esporas se observaron con un microscopio óptico con contraste de interferencia de Nomarski (Nikon Optiphot-II); los tamaños de las esporas, diámetros de las hifas y grosor de las capas de la pared se obtuvieron con una reglilla micrométrica acoplada a uno de los oculares. La coloración se obtuvo con la fórmula de porcentajes de azul, magenta, amarillo y negro propuesta por el INVAM (2013).

Las especies de HMA que se registran y describen en este trabajo pertenecen a los géneros Diversispora Walker y Schüßler, Gigaspora (Gerd. y Trappe) Walker y Sanders y Glomus Tul. y C. Tul. Los ejemplares de referencia están depositados en el Laboratorio de Biosistemática de Leguminosas, Departamento de Biología, Universidad Autónoma Metropolitana-Iztapalapa, y en el herbario del Centro de Investigación en Ciencias Biológicas (TLXM), Universidad Autónoma de Tlaxcala, México.

Diversispora trimurales (Koske y Halvorson) C. Walker y Schüßler (fig. 1). Basiónimo: Glomus trimurales Koske y Halvorson (1989) Mycologia 81(6): 927-933.

Esporas individuales y no en grupos en el suelo, de color amarillo claro (0/0/40/0), globosas, de 105-142 $\mu \mathrm{m}$ de diámetro. Pared de la espora compuesta por 3 capas, la más externa (L1) es amarillo claro (0/0/40/0), semipermanente, con una superficie irregular con proyecciones en forma de ámpulas que miden de 0.7-4 $\mu \mathrm{m}$ de alto; esta capa se pierde con facilidad. La capa intermedia (L2) es de color naranja brillante (0/0/80/0), permanente, rugosa, de $0.9 \mu \mathrm{m}$ de grosor. La capa más interna (L3) es hialina (0/0/10/0), laminada, lisa, de 4-7 $\mu \mathrm{m}$ de grosor, y está compuesta por capas muy delgadas que pueden separarse una de otra al aplicar presión. Ninguna capa presenta reacción con Melzer. La hifa de origen de la espora es hialina, cilíndrica, de $5 \mu \mathrm{m}$ de ancho en la base de la espora, su pared está compuesta por 3 capas continuas con las de la pared de la espora.

Diversispora trimurales difiere de otras especies de HMA que constituyen este género por el número y características de las capas que componen la pared de las esporas. Por ejemplo, Diversispora eburnea (Kenn., Stutz y Morton) Walker y Schüßler, D. epigea (Daniels y Trappe) Walker y Schüßler y $D$. spurca (Pfeiff., Walker y Bloss) Walker y Schüßler presentan solo 2 estratos (L1 y L2) y no 3 como en $D$. trimurales; mientras que D. aurantia (Blaszk. Blanke, Renker y Buscot) Walker y Schüßler, $D$. celata Walker, Gamper y Schüßler y $D$. clara Oehl, Estrada, Silva y Palenzuela también cuentan con 3 estratos; pero en estas especies de HMA la capa L3 es flexible y no laminada como en $D$. trimurales. Diversispora trimurales se registró por primera vez de la rizosfera de Abronia maritima Nutt. ex S. Wats. (Nyctaginaceae) y Ambrosia chamissonis (Less.) Greene (Asteraceae), ambas plantas de dunas costeras en los Estados Unidos de América (Koske y Halvorson, 1989). 


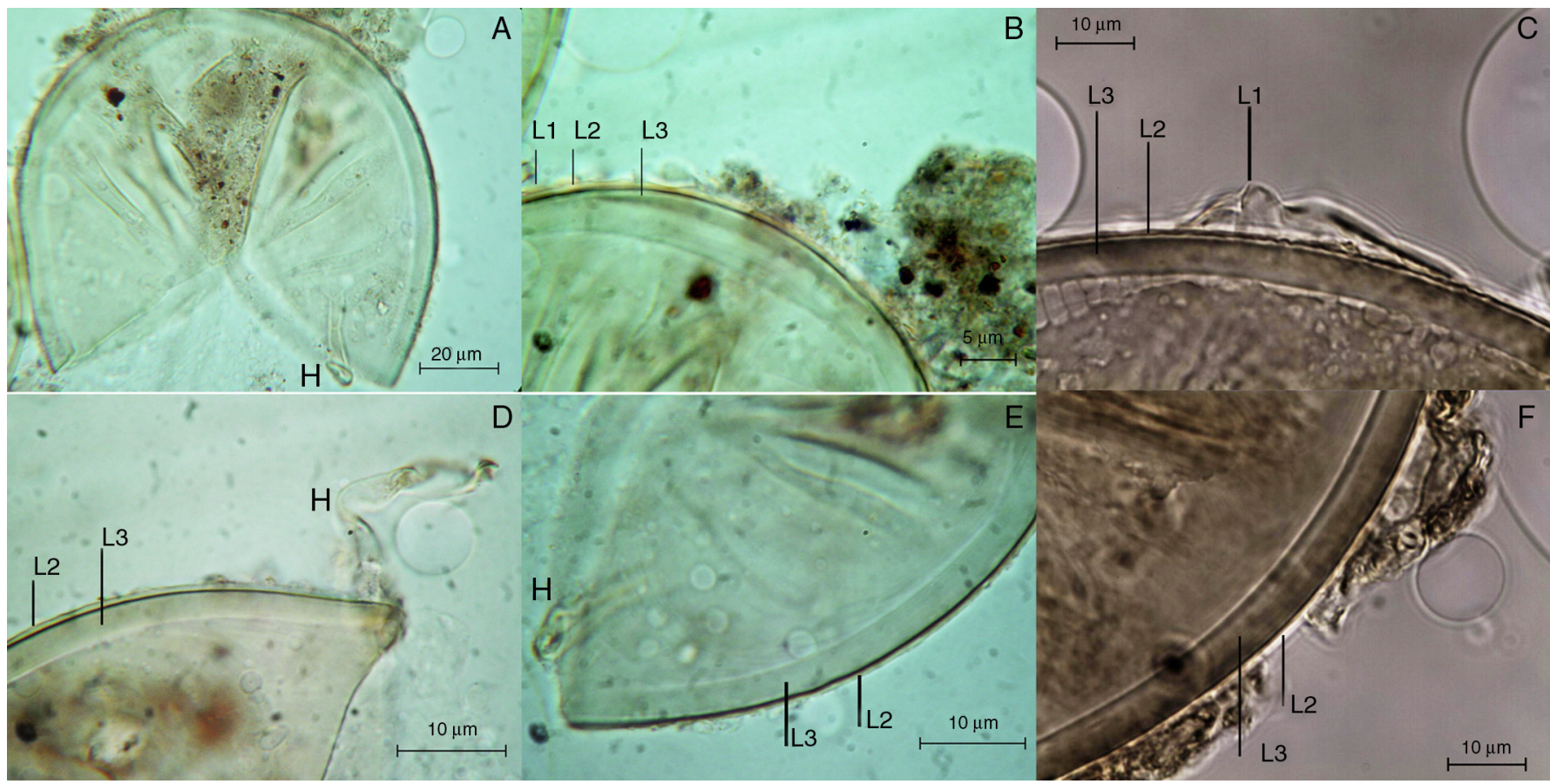

Figura 1. Esporas de Diversispora trimurales. A. Espora en alcohol polivinílico-lacto-glicerol con detalle de su hifa (H). B y C. Detalle de las capas externa (L1), intermedia (L2) e interna (L3) que componen la pared de las esporas. D. Espora en reactivo de Melzer, sin reacción de las capas L2 y L3. E. Detalle de las capas de la pared L2 y L3 e hifa. F. Contraste de las capas L2 y L3 de la pared con luz polarizada.

\section{Ejemplares examinados}

México, Puebla, municipio Chapulco, Loc. Azumbilla: km 68 de la carretera 469 Azumbilla-La Esperanza $\left(18^{\circ} 41^{\prime} 31^{\prime \prime} \mathrm{N}\right.$, 97²4'01.3”O), 2,291 m snm, 19/IV/2013, extraída de la rizosfera $(\mathrm{pH}=7.7 ; \mathrm{MO}=14.04 \% ; \mathrm{Nt}=0.6 \%$ y $\mathrm{Pi}=9 \mathrm{ppm})$ de M. lacerata $(\mathrm{Ml})$ y M. purpusii $(\mathrm{Mp})$. Los ejemplares se registraron únicamente en la estación seca, con una abundancia entre 19 y 25 esporas en $100 \mathrm{~g}$ de suelo. Claves de las preparaciones en el Laboratorio de Biosistemática de Leguminosas: Ml-063, Ml-065, Ml-066, Ml-069, Ml-070 y Mp-072 y en el herbario TLXM: TLXM6816.

Gigaspora candida Bhattacharjee, Mukerji, J.P. Tewari y Skoropad Trans. Br. Mycol. Soc. 78(1): 184-188 (fig. 2). Sinónimo: Gigaspora alboaurantiaca Wen-Neng, Chiang-Her y Hsu-Ho (1991) Trans. Mycol. Soc. ROC 6: 1-17.

Esporas individuales y no en grupos en el suelo, de color blanco con tonos de color amarillo (0/0/40/0) a naranja (0/10/100/0), globosas, de 200 a $250 \mu \mathrm{m}$ de diámetro. La pared de la espora está compuesta de 3 capas: la más externa (L1) es hialina, rígida, permanente, lisa, de 0.7-1.0 $\mu \mathrm{m}$ de grosor; la capa intermedia (L2) es de color naranja (0/10/100/0), laminada, permanente, lisa, de $13.3 \mu \mathrm{m}$ de grosor, cambia a una coloración café-rojizo oscuro (60/80/100/10) con Melzer; la capa interna (L3) es de color naranja (0/10/100/0), permanente, está fuertemente adherida a la L2, por lo que es difícil de observar, solo es detectable cuando hay papilas germinales. La hifa de origen es en forma de bulbo, de $48.2 \mu \mathrm{m}$ de ancho en promedio.

Gigaspora candida es diferente de otras especies del género que generalmente forman esporas de un tamaño mayor a $300 \mu \mathrm{m}$
(Oehl, de Souza y Sieverding, 2008), como G. decipiens Hall y Abbott, cuyo color varía de blanco a crema $(0 / 5 / 30 / 0)$ y la capa L2 mide más de $25 \mu \mathrm{m}$ de grosor; G. gigantea (Nicol. y Gerd.) Gerd y Trappe, que presenta un color verde (20/0/100/0) asociado al contenido de las esporas; G. margarita Becker y Hall y G. ramisporophora Spain, Sieverd y Schenck, ambas de color crema (0/5/30/0), la primera con L2 midiendo en promedio $22 \mu \mathrm{m}$ de grosor y la última especie con varios bulbos en la hifa. Gigaspora candida es similar en tamaño a G. rosea Nicolson y Schenck, pero esta última presenta una coloración blanca con tintes rosados $(0 / 5 / 10 / 0)$ y su bulbo es más oscuro que la pared de la espora. Por último, G. albida Schenck y Sm. es de color crema con tintes verdosos $(5 / 0 / 20 / 0)$ y su pared compuesta por 3 capas (L1, L2 y L3), con L2 midiendo entre 15 y $26 \mu \mathrm{m}$ de grosor. Gigaspora candida se registró por primera vez en suelos con cultivo de trigo en Wazirabad, India (Bhattacharjee, Mukerji, Tewari y Skoropad, 1982).

\section{Ejemplares examinados}

México, Puebla, municipio Coxcatlán, $1 \mathrm{~km}$ hacia el sur del poblado de Coxcatlán, carretera 980 Coxcatlán-Teotitlán de Flores Magón, 18¹5'23.7” N, 9709'03.3” O, 1,140 m snm, 06/X/2012 y 19/IV/2013; se registró en la rizosfera $(\mathrm{pH}=6.0$; $\mathrm{MO}=2.9 \% ; \mathrm{Nt}=0.15 \%$ y $\mathrm{Pi}=10 \mathrm{ppm}$ ) de $M$. luisana (Mluis) y M. polyantha (Mpoly), y en suelo de áreas abiertas (AA), sin vegetación $(\mathrm{pH}=6.7 ; \mathrm{MO}=1.6 \% ; \mathrm{Nt}=0.08 \%$ y $\mathrm{Pi}=5.5 \mathrm{ppm})$. Los ejemplares se registraron en ambas estaciones de muestreo cuando se trató del suelo de la rizosfera, pero en AA solo se registraron en la estación seca; su abundancia de esporas osciló entre 31 y 47 por $100 \mathrm{~g}$ de suelo. Claves de las preparaciones en el Laboratorio de Biosistemática de Leguminosas: Mluis-033, 


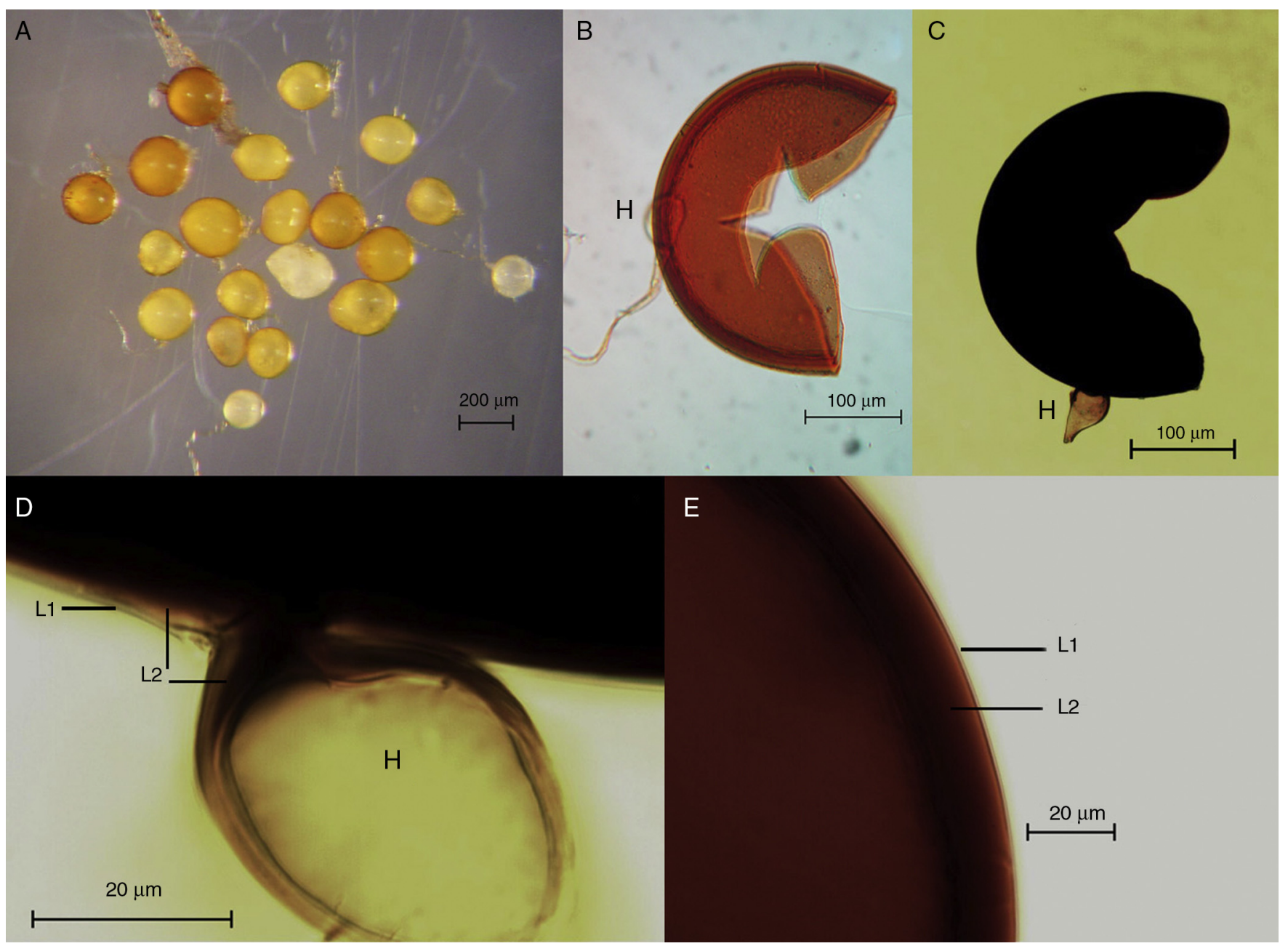

Figura 2. Esporas de Gigaspora candida. A. Esporas en agua. B. Espora en alcohol polivinílico-lacto-glicerol con detalle de su hifa en forma de bulbo (H). C. Espora con reacción al reactivo de Melzer. D. Detalle de las capas externa L1 e intermedia L2 de la pared que son continuas con la pared de la hifa (H). E. Capas de la pared de la espora con reacción al reactivo de Melzer en la capa L2.

Mluis-035 a 037, Mluis-091 a 098; Mpoly-042 a 043, Mpoly045 a 048, Mpoly-102 a 103, Mpoly-105 a 107; AA-117 y AA-119 y en el herbario TLXM: TLXM6808 y TLXM6809.

\section{Glomus corymbiforme Blaszkowski Mycologia 87(5):} 732-737 (fig. 3).

Esporas en agregados en forma de corimbos, producidas a partir de una hifa primaria denominada esporóforo, cenocítica, cilíndrica, de 9-13 $\mu \mathrm{m}$ de ancho, su pared es de color naranja (0/30/100/0), de 0.6-1.9 $\mu \mathrm{m}$ de grosor, se ramifica dicotómicamente y forma esporas en los ápices, en donde la pared de la hifa tiene mayor grosor y varía de 2.2-3.7 $\mu \mathrm{m}$. Peridio hialino, constituido por una red de hifas que envuelven a las esporas, parcial o totalmente ausente en esporas maduras. Las esporas son de color naranja (0/30/100/0), globosas, de 50-65 $\mu \mathrm{m}$ de diámetro. La pared de la espora está constituida por 3 capas continuas con la pared de la hifa. La más externa (L1) es hialina, permanente, rígida, lisa, de 0.9-1.5 $\mu \mathrm{m}$ de grosor; la capa intermedia (L2) es de color naranja (0/30/100/0), laminada, de 2-3 $\mu \mathrm{m}$ de grosor; y la capa interna (L3) es hialina, semiflexible, de 0.5-1 $\mu \mathrm{m}$ de grosor, esta capa está ligeramente adherida a la L2 y forma un septo cerca de la base de la espora. Ninguna de las capas de la pared esporal o del peridio hifal reacciona con Melzer.

Las características morfológicas de $G$. corymbiforme permiten distinguirla con claridad de otras especies de HMA del orden Glomerales que forman esporocarpos con peridio, como Funneliformis mosseae (Nicolson y Gerd.) Walker y Schüßler, G. convolutum Gerd. y Trappe y G. mortonii Bentiv. y Hetrick, en donde la capa L1 de la pared de las esporas reacciona al Melzer; además, en la última especie, la capa L3 es laminada. Asimismo, difiere de G. pubescens (Sacc. y Ellis) Höhn que tiene esporas hialinas muy pequeñas $(25-36 \mu \mathrm{m}) ;$ G. tortuosum Schenck y Smith cuyas esporas presentan solo una capa laminada, y de $G$. globiferum Koske y Walker que tiene un peridio con vesículas y no forma esporocarpos. Glomus corymbiforme se registró por primera vez de la rizosfera de Ammophila arenaria (L.) Link. (Poaceae), planta que habita en las dunas marítimas de Swinojscie, al noroeste de Polonia (Blaszkowski, 1995).

\section{Ejemplares examinados}

México, Puebla, municipio Coxcatlán, a $1 \mathrm{~km}$ hacia el sur del poblado de Coxcatlán, carretera 980 Coxcatlán-Teotitlán 


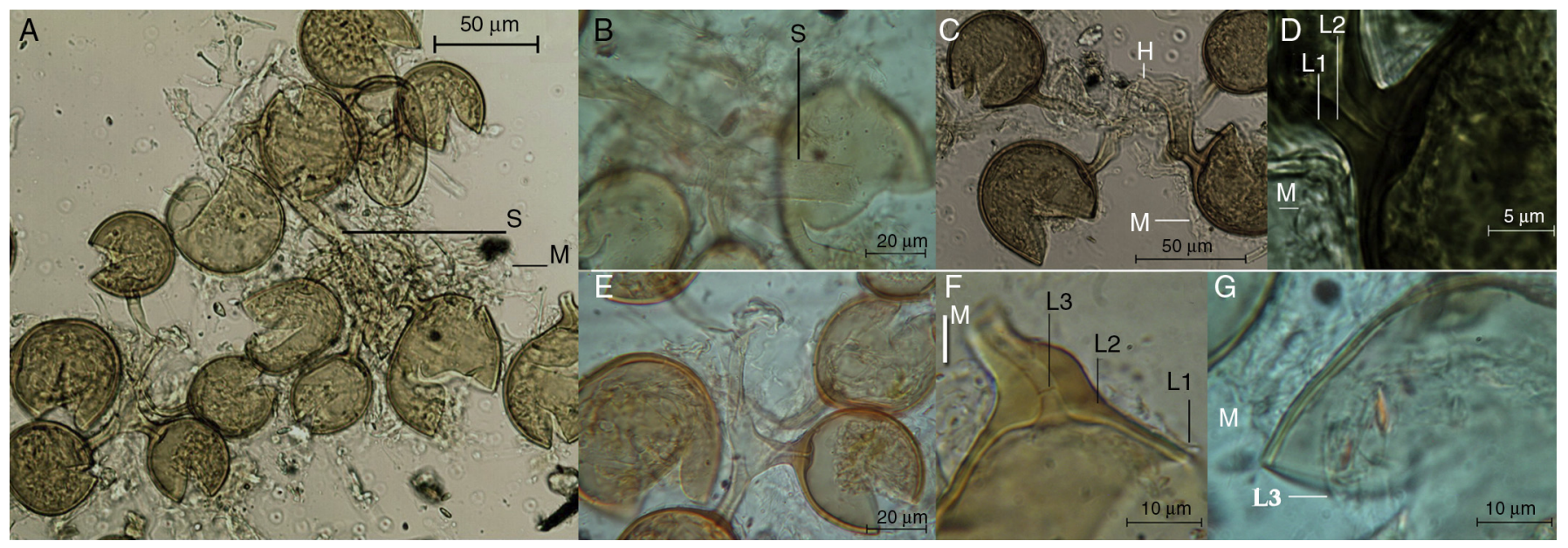

Figura 3. Esporas de Glomus corymbiforme con reactivo Melzer. A. Esporocarpo mostrando el esporóforo (S) y restos del manto hifal (M). B. Detalle del esporóforo. C. Esporas con manto hifal. D. Detalle de las capas externa (L1) e intermedia (L2) de la hifa que son continuas con la pared de la espora. E. Detalle del arreglo dicotómico de las esporas. F. Detalle de las capas L1, L2 e interna (L3) de la pared esporal con la capa L3, formando el septo en la hifa. G. Detalle del manto hifal y de la capa L3 que es hialina y semiflexible.

de Flores Magón, $18^{\circ} 15^{\prime} 23.7^{\prime \prime} \mathrm{N}, 97^{\circ} 09^{\prime} 03.3$ ” O, 1,140 m snm, $06 / \mathrm{X} / 2012$; se presentó en la rizosfera $(\mathrm{pH}=6.0 ; \mathrm{MO}=2.7 \%$; $\mathrm{Nt}=0.14 \%$ y Pi $=9.5 \mathrm{ppm}$ ) de M. luisana (Mluis). Los ejemplares se registraron únicamente en la estación de lluvias con una abundancia de 21 esporas en $100 \mathrm{~g}$ de suelo. Claves de las preparaciones en el Laboratorio de Biosistemática de Leguminosas: Mluis-035 y en el herbario TLXM: TLXM6806.

En el Valle de Tehuacán-Cuicatlán las especies endémicas de Mimosa son un componente importante en la estructura de la vegetación de los matorrales xerófilos, y son elementos relevantes para el funcionamiento de los ecosistemas semiáridos al formar islas de recursos y contribuir a la fertilidad del suelo (Camargo-Ricalde y Dhillion, 2003; Camargo-Ricalde et al., 2002; Camargo-Ricalde, Reyes-Jaramillo y Montaño, 2010). Los 3 nuevos registros de HMA que se documentan para México están ligados a 4 especies de Mimosa. Diversispora trimurales se registró en la rizosfera de $M$. lacerata (25\% de las muestras) y de M. purpusii (10\%); G. corymbiforme solo en la rizosfera de $M$. luisana (10\%), y G. candida se presentó en la rizosfera de M. luisana $(60 \%)$ y de $M$. polyantha (55\%), así como en áreas abiertas (10\%), indicando que la formación de esporas en esta especie de HMA podría también ocurrir en condiciones con mayor estrés ambiental y no restringirse al suelo asociado a la rizosfera de las especies de Mimosa en este ecosistema semiárido. Las diferencias en abundancias de esporas entre las especies descritas y su presencia o ausencia, en relación con la estacionalidad de la lluvia, sugiere posibles variaciones fenológicas en la formación de esporas (Pringle y Bever, 2002) que podrían estar relacionadas con cambios en la humedad del suelo, como se registra en zonas desérticas (Allen, 1983). Asimismo, la presencia de nuevos registros resalta la importancia de los taxa de Mimosa endémicas como reservorios de la riqueza de especies de HMA. Con estos 3 nuevos registros de HMA se incrementa el número de especies conocidas de Glomeromycota para México a 104, lo que representa el $44 \%$ de las especies descritas en el mundo (Oehl, Sieverding, Palenzuela, Ineichen y Gladstone, 2011; Schüßler y Walker, 2010).
El Valle de Tehuacán-Cuicatlán es reconocido por su alta diversidad de plantas y endemismos (Dávila et al., 2002), y como una ecorregión prioritaria para la conservación de la biodiversidad, por lo que se requiere de mayor cantidad de estudios enfocados a documentar la abundancia, diversidad y riqueza de diferentes grupos microbianos, entre ellos los simbióticos mutualistas, así como su papel ecológico y funcional. Sobre todo, porque se desconoce la identidad de los HMA asociados con la mayor parte de las plantas dominantes, endémicas o emblemáticas de la zona, como es el caso de las especies del género Mimosa, las cuales pueden ser un componente vegetal esencial para la conservación y promoción in situ de la biodiversidad de HMA.

Finalmente, y desde una perspectiva global para México, los 3 nuevos registros de HMA recolectados del suelo rizosférico de 4 de las 16 taxa de Mimosa documentadas para el Valle de Tehuacán-Cuicatlán (Martínez-Bernal y Grether, 2006), sugieren la posibilidad de que otras especies de HMA, aún no registradas para México e incluso poco conocidas a nivel mundial, estén presentes en la rizosfera de los 12 taxa de Mimosa restantes, así como en otras taxa de Mimosa que se establecen en el país ( $c a$. 110 especies, 60\% endémicas para México; Grether, Camargo-Ricalde y Martínez-Bernal, 1996). Es importante mencionar que en este trabajo solo está representado el $3.64 \%$ de las especies de Mimosa conocidas en México, por lo que se requiere dirigir este tipo de estudios hacia otros taxa de Mimosa presentes en los ecosistemas, principalmente áridos y semiáridos del país, ya que además de desconocerse el estatus micorrícico de estos taxa, también se desconoce la abundancia, diversidad y riqueza de Glomeromycota que albergan. Asimismo, es recomendable realizar cultivos monoespecíficos de las especies de HMA aquí descritas, con la finalidad de contribuir a su conservación ex situ.

A Andrea Gutiérrez-Ponce y a Luis Pedroza por su apoyo en el trabajo de campo, a la Maestría en Biología, Universidad Autónoma Metropolitana-Iztapalapa y al Consejo Nacional de Ciencia y Tecnología (Conacyt) por la beca otorgada a Eduardo 
Chimal Sánchez (Beca Núm. 283809) para sus estudios de Posgrado.

\section{Referencias}

Allen, M. F. (1983). Formation of vesicular-arbuscular mycorrhizae in Atriplex gardneri (Chenopodiaceae): seasonal response in a cold desert. Mycologia, $75,773-776$.

Bhattacharjee, M., Mukerji, G. K., Tewari, P. J. y Skoropad, P. E. (1982). Structure and hyperparasitism of a new species of Gigaspora. British Mycological Society, 78, 184-188.

Blaszkowski, J. (1995). Glomus corymbiforme, a new species in Glomales from Poland. Mycologia, 87, 732-737.

Brundrett, C. M. (2009). Mycorrhizal associations and other means of nutrition of vascular plants: Understanding the global diversity of host plants by resolving conflicting information and developing reliable means of diagnosis. Plant and Soil, 320, 37-77.

Camargo-Ricalde, S. L. y Dhillion, S. S. (2003). Endemic Mimosa species can serve as mycorrhizal "resource islands" within semiarid communities of the Tehuacán-Cuicatlán Valley, Mexico. Mycorrhiza, 13, 129-136.

Camargo-Ricalde, S. L., Dhillion, S. S. y Grether, R. (2002). Community structure of endemic Mimosa species and environmental heterogeneity in a semi-arid Mexican valley. Journal of Vegetation Science, 13, 697-704.

Camargo-Ricalde, S. L., Reyes-Jaramillo, I. y Montaño, N. M. (2010). Forestry insularity effect of four Mimosa L. species (Leguminosae-Mimosoideae) on soil nutrients of a Mexican semiarid ecosystem. Agroforestry Systems, 80, 385-397.

Camargo-Ricalde, S. L. y Esperón-Rodríguez, M. (2005). Efecto de la heterogeneidad espacial y temporal del suelo sobre la abundancia de esporas de hongos micorrizógenos arbusculares en el Valle de Tehuacán-Cuicatlán, México. Revista de Biología Tropical, 53, 339-352.

Dávila, P., Arizmendi, M. C., Valiente-Banuet, A., Villaseñor, J. L., Casas, A. y Lira, R. (2002). Biological diversity in the Tehuacán-Cuicatlán Valley, Mexico. Biodiversity and Conservation, 11, 421-442.

Gerdemann, J. W. y Nicolson, H. T. (1963). Spores of mycorrhizal Endogone species extracted from soil by wet sieving and decanting. British Mycological Society, 46, 235-244.

Grether, R., Camargo-Ricalde, S. L. y Martínez-Bernal, A. (1996). Especies del género Mimosa (Leguminosae) en México. Boletín de la Sociedad Botánica de México, 58, 149-152.

INVAM (International Culture Collection of [Vesicular] Arbuscular Mycorrhizal Fungi). (2013) [consultado 20 Feb 2014]. Disponible en: http://invam.caf. wvu.edu/
Koske, E. R. y Halvorson, L. W. (1989). Scutellospora arenicola and Glomus trimurales: two new species in the Endogonaceae. Mycologia, 81, 927-933.

Maherali, H. y Klironomos, N. J. (2007). Influence of phylogeny on fungal community assembly and ecosystem functioning. Science, 316, 1746-1748.

Martínez-Bernal, A. y Grether, R. (2006). Mimosa L. En A. Novelo y R. MedinaLemus (Eds.), Flora del Valle de Tehuacán-Cuicatlán. (pp. 42-99). México, D.F: Instituto de Biología, Universidad Nacional Autónoma de México.

Méndez-Cortés, H., Marmolejo, M. J. G., Olalde-Portugal, V., Cantú, A. C. M. y Varela, L. (2012). Nuevos registro de hongos micorrizógenos arbusculares para México. Revista Mexicana de Micología, 36, 49-56.

Montaño, N. M., Alarcón, A., Camargo-Ricalde, S. L., Hernández-Cuevas, L. V., Álvarez-Sánchez, J., González-Chávez, M. C., et al. (2012). Research on arbuscular mycorrhizae in Mexico: an historical synthesis and future prospects. Symbiosis, 57, 111-126.

Oehl, F., De Souza, A. F. y Sieverding, E. (2008). Revision of Scutellospora and description of five new genera and three new families in the arbuscular mycorrhiza-forming Glomeromycetes. Mycotaxon, 106, 311-360.

Oehl, F., Sieverding, E., Palenzuela, J., Ineichen, K. y Gladstone, S. A. (2011). Advances in Glomeromycota taxonomy and classification. International Mycological Association Fungus, 2, 191-199.

Pringle, A. y Bever, D. J. (2002). Divergent phenologies may facilitate the coexistence of arbuscular mycorrhizal fungi in a North Carolina grassland. American Journal of Botany, 89, 1439-1446.

Schüßler, A., Schwarzott, D. y Walker, C. (2001). A new fungal phylum, the Glomeromycota: phylogeny and evolution. Mycological Research, 105, 1413-1421.

Schüßler, A. y Walker, C. (2010). The Glomeromycota: a species list with new families and new genera [consultado 2 Feb 2014]. Disponible en: http://schuessler.userweb.mwn.de/amphylo/.

Semarnat (Secretaría de Medio Ambiente y Recursos Naturales). (1998). Decreto por el que se declara área natural protegida con el carácter de reserva de la biosfera, la región denominada Tehuacán-Cuicatlán, ubicada en los estados de Oaxaca y Puebla, Estados Unidos Mexicanos, Presidencia de la República. Diario Oficial de la Federación. 18 de Sep de 1998, México, D.F.

Smith, E. S., Facelli, E., Pope, S. y Smith, F. A. (2010). Plant performance in stressful environments: interpreting new and established knowledge of the roles of arbuscular mycorrhizas. Plant and Soil, 326, 3-20.

Van der Heijden, A. M. G., Klironomos, N. J., Ursic, M., Moutoglis, P., Streitwolf-Engel, R., Boller, T., et al. (1998). Mycorrhizal fungal diversity determines plant biodiversity, ecosystem variability and productivity. Nature, 396, 69-72.

Wen-Neng, Ch., Chiang-Her, Y. y Hsu-Ho, C. H. (1991). Species of Gigaspora and Scutellospora (endogonaceae) in Taiwan. Transactions Mycological Society, 6, 1-17. 